\title{
国圈 CAMBRIDGE
}

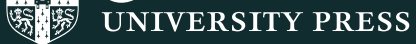

